\title{
THE DE BRANGES THEOREM ON UNIVALENT FUNCTIONS ${ }^{1}$
}

\author{
BY
}

\section{CARL H. FITZGERALD AND CH. POMMERENKE}

\begin{abstract}
We present a simplified version of the de Branges proof of the LebedevMilin conjecture which implies the Robertson and Bieberbach conjectures. As an application of an analysis of the technique, it is shown that the method could not be used directly to prove the Bieberbach conjecture.
\end{abstract}

1. Introduction. Let $S$ denote the class of all functions

$$
f(z)=z+\sum_{n=2}^{\infty} a_{n} z^{n}
$$

that are analytic and univalent in the unit disk $\mathscr{D}$. Louis de Branges recently proved [3-5] the following inequality which implies the Bieberbach conjecture.

De Branges Theorem. Suppose $f \in S$ and define $c_{k}$ :

$$
\log \frac{f(z)}{z}=\sum_{k=1}^{\infty} c_{k} z^{k} \text { for } z \text { in } \mathscr{D} .
$$

Then, for $n=1,2, \ldots$,

$$
\sum_{k=1}^{n} k(n+1-k)\left|c_{k}\right|^{2} \leqslant 4 \sum_{k=1}^{n} \frac{n+1-k}{k} .
$$

The inequality (1.3) was conjectured by N. A. Lebedev and I. M. Milin [10] in 1971. The Lebedev-Milin exponentiation inequality [9, 10] (see e.g. [11, Lemma 3.3]) shows inequality (1.3) implies a conjecture made by M. S. Robertson [12] in 1936: If $f$ is an odd function in $S$, then

$$
\sum_{k=1}^{m}\left|a_{2 k-1}\right|^{2} \leqslant m \text { for } m=1,2, \ldots
$$

An elementary application of inequality (1.4) to the odd function $\sqrt{f\left(z^{2}\right)}$ proves a conjecture made by L. Bieberbach [2] in 1916: If $f$ is a function in $S$, then

$$
\left|a_{n}\right| \leqslant n \text {. }
$$

Received by the editors October 16, 1984 and, in revised form, November 13, 1984.

1980 Mathematics Subject Classification. Primary 30C50; Secondary 30C75.

${ }^{1}$ This work was supported in part by the National Science Foundation. 
Furthermore, Robertson [13] (see e.g. [11, Corollary 2.2]) has shown that inequality (1.4) proved by de Branges has the following consequence.

COROllaRY. If $f(z)=a_{1} z+a_{2} z^{2}+\cdots$ satisfies

$$
|f(z)| \leqslant|g(\varphi(z))|, \quad \text { where }|\varphi(z)| \leqslant|z|,
$$

for $z \in \mathscr{D}$ and $g \in S$, then (1.5) holds.

In short, de Branges has proved the Bieberbach conjecture in its most general form. The proof was announced by de Branges in lectures he gave in the Leningrad geometric function theory seminar. A distillation of his argument by E. G. Emel'anov, G. V. Kuz'mina and I. M. Milin was circulated; he published a similar version [3]. A more formal presentation is to appear [4].

The proof of de Branges is surprisingly short in light of the great effort that has gone into trying to prove the Bieberbach conjecture. His argument [3] was made in two steps. First he proves a more general result on bounded univalent functions by the ordinary Löwner differential equation which describes a contracting flow on the unit disk. Then he applies this result to prove his inequality (1.3).

We shall give an even shorter version of that proof by making a technical change. We will use the linear partial differential equation of Löwner that describes an expanding flow in the $\mathrm{p}^{\mathrm{l}} \mathrm{dne}$. One result is that certain approximations in the first step can be dispensed with. De Branges independently makes a similar change in the proof [4].

We can also settle the case of equality. (With some effort, the same conclusion follows from de Branges's method of proof [3], and the result appears explicitly in [4].)

THEOREM. If $f \in S$ and

$$
f(z) \not \equiv \frac{z}{(1-\zeta z)^{2}} \quad \text { for some } \zeta \in \mathbf{C} \text { with }|\zeta|=1,
$$

then strict inequality holds in (1.3).

It follows that if $f \in S$ and inequality (1.7) holds, then $\left|a_{n}\right|<n$ for $n=2,3, \ldots$, that is, the only functions of $S$ for which equality holds in the Bieberbach estimate for some $n$ are rotations of the Koebe function.

Finally we make some remarks about the proof. It is pointed out that the more general inequality of de Branges follows by a slight change of our proof. Then the choice of weight functions is motivated. Since they are essentially unique, it becomes possible to ask whether the de Branges method of proof could be directly applied to the Bieberbach conjecture. The answer shows the important role of the Lebedev-Milin conjecture.

2. The special function system of de Branges. To avoid interrupting the proof, we will first make some observations about a system of functions introduced by de Branges.

Fix a positive integer $n$. For $k=1,2, \ldots, n$, let

$$
\tau_{k}(t)=k \sum_{\nu=0}^{n-k}(-1)^{\nu} \frac{(2 k+\nu+1)_{\nu}(2 k+2 \nu+2)_{n-k-\nu}}{(k+\nu) \nu !(n-k-\nu) !} e^{-(\nu+k) t}
$$


where $(a)_{\nu}$ denotes $a(a+1)(a+2) \cdots(a+\nu-1)$. Let $\tau_{n+1}(t) \equiv 0$. Direct calculation shows

$$
\tau_{k}(t)-\tau_{k+1}(t)=-\frac{\tau_{k}^{\prime}(t)}{k}-\frac{\tau_{k+1}^{\prime}(t)}{k+1}
$$

Let $P_{j}(\alpha, \beta)$ denote the Jacobi polynomials (see e.g. [14]). It is easily deduced [1, p. 717] from (2.1) that

$$
\tau_{k}^{\prime}(t)=-k e^{-k t} \sum_{j=0}^{n-k} P_{j}^{(2 k, 0)}\left(1-2 e^{-t}\right) .
$$

Since $P_{j}^{(\alpha, 0)}(-1)=(-1)^{j}\left[14\right.$, p. 59], it follows that $\tau_{k}^{\prime}(0)=-k$ if $n-k$ is even and $\tau_{k}^{\prime}(0)=0$ if $n-k$ is odd. Hence equality (2.2) implies $\tau_{k}(0)-\tau_{k+1}(0)=1$; and descending induction shows that

$$
\tau_{k}(0)=n+1-k
$$

It follows from (2.3) by the result of Askey and Gaspar [1, Theorem 3] that

$$
\tau_{k}^{\prime}(t)<0 \text { for } 0<t<+\infty .
$$

Gasper [7] has recently given a different proof of inequality (2.5) where (2.3) is formulated in terms of generalized hypergeometric functions instead of Jacobi polynomials.

3. Proof of the de Branges Theorem. In 1923 Löwner [8] (e.g. [6, Chapter 3]) proved the following representation theorem: If $f$ is a function in $S$ such that

$$
f(\mathscr{D})=\mathbf{C} \backslash J, \quad \text { where } J \text { is a Jordan arc entending to infinity, }
$$

then there is a parametrized family of univalent functions

$$
f(z, t)=e^{t} z+\cdots \quad \text { for } z \in D \text { and } 0 \leqslant t<+\infty
$$

such that $f(z, 0)=f(z)$ and

$$
\frac{\partial}{\partial t} f(z, t)=\frac{1+\kappa(t) z}{1-\kappa(t) z} z \frac{\partial}{\partial z} f(z, t),
$$

where $|\kappa(t)|=1$ and $\kappa(t)$ is a continuous function on $[0, \infty)$. The functions $f$ that satisfy (3.1) are dense in $S$ with respect to uniform convergence on compact subsets of $\mathscr{D}$. Hence it is sufficient to prove inequality (1.3) for these functions.

For $0 \leqslant t<\infty$ define $c_{k}(t)$ by

$$
\log \frac{f(z, t)}{e^{t} z}=\sum_{k=1}^{\infty} c_{k}(t) z^{k} \quad \text { for } z \in \mathscr{D}
$$

so that $c_{k}(0)=c_{k}$ by (1.2). The following equations are obtained by the application of $\partial / \partial t$ to (3.4), the use of (3.3) and the application of $\partial / \partial z$ to (3.4):

$$
\begin{aligned}
1+\sum_{k=1}^{\infty} c_{k}^{\prime}(t) z^{k} & =\frac{1+\kappa(t) z}{1-\kappa(t) z} z\left[\frac{\partial}{\partial z} f(z, t)\right] / f(z, t) \\
& =\left(1+2 \sum_{k=1}^{\infty} \kappa(t)^{k} z^{k}\right)\left(1+\sum_{k=1}^{\infty} k c_{k}(t) z^{k}\right) .
\end{aligned}
$$


From (3.5) we obtain

$$
c_{k}^{\prime}(t)=2 \sum_{j=1}^{k-1} j c_{j}(t) \kappa(t)^{k-j}+k c_{k}(t)+2 \kappa(t)^{k}
$$

Let $b_{0}(t)=0$ and

$$
b_{k}(t)=\sum_{j=1}^{k} j c_{j}(t) \kappa(t)^{-j}
$$

for $k=1,2, \ldots$ Equation (3.6) can be simplified to

$$
c_{k}^{\prime}(t)=\kappa(t)^{k}\left[b_{k}(t)+b_{k-1}(t)+2\right] .
$$

Let $n$ be a fixed positive integer. Define

$$
\varphi(t)=\sum_{k=1}^{n}\left(k\left|c_{k}(t)\right|^{2}-\frac{4}{k}\right) \tau_{k}(t) \text { for } 0 \leqslant t<+\infty .
$$

We now suppress the variable $t$. By (3.7), $k \bar{c}_{k}=\left(\bar{b}_{k}-\bar{b}_{k-1}\right) \bar{\kappa}^{k}$. By using (3.8) and differentiating (3.9), we conclude that

$$
\begin{aligned}
\varphi^{\prime}= & \sum_{k=1}^{n} 2 \operatorname{Re}\left[\left(\bar{b}_{k}-\bar{b}_{k-1}\right)\left(b_{k}+b_{k-1}+2\right)\right] \tau_{k} \\
& +\sum_{k=1}^{n}\left(\left|b_{k}-b_{k-1}\right|^{2}-4\right) \tau_{k}^{\prime} .
\end{aligned}
$$

Note $\operatorname{Re}[]=\left|b_{k}\right|^{2}-\left|b_{k-1}\right|^{2}+2 \operatorname{Re} b_{k}-2 \operatorname{Re} b_{k-1}$, and recall $b_{0}=0$ and $\tau_{n+1}=0$. By partial summation we obtain

$$
\varphi^{\prime}=\sum_{k=1}^{n}\left(2\left|b_{k}\right|^{2}+4 \operatorname{Re} b_{k}\right)\left(\tau_{k}-\tau_{k+1}\right)+\sum_{k=1}^{n}\left(\left|b_{k}-b_{k-1}\right|^{2}-4\right) \frac{\tau_{k}^{\prime}}{k}
$$

Now it follows from the differential equation (2.2) that the first summation in (3.10) is

$$
\begin{aligned}
& -\sum_{k=1}^{n}\left(2\left|b_{k}\right|^{2}+4 \operatorname{Re} b_{k}\right)\left(\frac{\tau_{k}^{\prime}}{k}+\frac{\tau_{k+1}^{\prime}}{k+1}\right) \\
& =-\sum_{k=1}^{n}\left(2\left|b_{k}\right|^{2}+4 \operatorname{Re} b_{k}+2\left|b_{k-1}\right|^{2}+4 \operatorname{Re} b_{k-1}\right) \frac{\tau_{k}^{\prime}}{k} .
\end{aligned}
$$

Hence we see from (3.10) that

$$
\varphi^{\prime}(t)=-\sum_{k=1}^{n}\left|b_{k-1}(t)+b_{k}(t)+2\right|^{2} \frac{\tau_{k}^{\prime}(t)}{k} .
$$

From inequality (2.5) we conclude

$$
\varphi^{\prime}(t) \geqslant 0 \text { for } 0 \leqslant t<+\infty .
$$

This inequality is the key.

Now we use that $e^{-t} f(z, t)$ belongs to $S$; (3.2) shows the normalization conditions are satisfied. Since $S$ is compact, if $k$ is fixed, $\left|c_{k}(t)\right|$ remains bounded as $t \rightarrow+\infty$. Also note that (2.1) implies that $\tau_{k}(t)$ tends to zero as $t$ tends to infinity for each $k$. From (3.9) it follows that $\varphi(+\infty)=0$, and from inequality (3.12),

$$
\sum_{k=1}^{n}\left(k\left|c_{k}(0)\right|^{2}-\frac{4}{k}\right)(n+1-k)=-\int_{0}^{\infty} \varphi^{\prime}(t) d t \leqslant 0
$$


Since $f(z, 0)=f(z)$, inequality (1.3) is proved for functions of $S$ satisfying (3.1) and therefore for all functions in $S$.

4. Equality. Now we prove the theorem stated in the first section. We assume inequality (1.7) holds. Then $\left|a_{2}\right|<2$ as Bieberbach [2] (see e.g. [11, Theorem 1.5]) has shown. We choose a sequence of functions $f_{m} \in S$ which satisfy (3.1) and converge to $f$ uniformly on compact subsets of $\mathscr{D}$. We add the subscript to the corresponding coefficients. From (1.1) and (1.2), for some number $\alpha$,

$$
\left|c_{1, m}\right|=\left|a_{2, m}\right|<\alpha<2
$$

at $t=0$ for large $m$.

The Löwner differential equation is used in the form given by (3.6). It follows that

$$
\left|c_{1, m}^{\prime}\right|=\left|c_{1, m}(t)+2 \kappa_{m}(t)\right| \leqslant\left|a_{2, m}(t)\right|+2 \leqslant 4 .
$$

Hence from (4.1), we see that $c_{1, m}(t) \leqslant \alpha+4 t$. From (3.10) and inequality (2.5),

$$
\varphi_{m}^{\prime}(t) \geqslant\left|c_{1, m}(t) \bar{\kappa}_{m}(t)+2\right|^{2}\left(-\tau_{1}^{\prime}(t)\right) \geqslant(2-\alpha-4 t)^{2}\left(-\tau_{1}^{\prime}(t)\right)
$$

for $0 \leqslant t \leqslant(2-\alpha) / 4$ and $m$ large. Hence by (3.13)

$$
\begin{aligned}
\sum_{k=1}^{n}\left(k\left|c_{k, m}\right|^{2}-\frac{4}{k}\right)(n+1-k) & \\
& \leqslant-\int_{0}^{(2-\alpha) / 8} \varphi_{m}^{\prime}(t) d t \leqslant\left(\frac{2-\alpha}{2}\right)^{2} \int_{0}^{(2-\alpha) / 8} \tau_{1}^{\prime}(t) d t \\
& =\left(\frac{2-\alpha}{2}\right)^{2}\left[\tau_{1}\left(\frac{2-\alpha}{8}\right)-\tau_{1}(0)\right]<0 .
\end{aligned}
$$

Letting $m$ tend to infinity, we conclude strict inequality holds in (1.3) and consequently in the Bieberbach estimate.

5. Remarks. (1) De Branges has proved a more general inequality; specifically, if $f \in S$ and $|f(z)|<e^{T}$, then

$$
\sum_{k=1}^{n} k(n+1-k)\left|c_{k}+q_{k}\right|^{2} \leqslant \sum_{k=1}^{n} k\left|p_{k}\right|^{2} \tau_{k}(T)+\sum_{k=1}^{n} \frac{4}{k}\left(n+1-k-\tau_{k}(T)\right),
$$

where the $p_{k}$ are arbitrary complex numbers and the $q_{k}$ are determined by

$$
\sum_{k=1}^{\infty} q_{k} z^{k}=\sum_{k=1}^{\infty} p_{k} e^{-k T} f(z)^{k}
$$

His inequality (1.3) follows by letting $p_{k}=0$ and $T$ tend to $\infty$. By a slight change in (3.4), our proof also gives inequality (5.1). Use the coefficients of the expansion of

$$
\log \frac{f(z, t)}{e^{t} z}+\sum_{k=1}^{\infty} p_{k} e^{-k T} f(z, t)^{k}
$$

to define $\varphi$. Integrate $-\varphi^{\prime} \leqslant 0$ from 0 to $T$.

(2) The choice of the weight functions $\tau_{k}$ made by de Branges can be motivated and shown to be unique in a certain sense. 
For the Koebe function $f(z)=z(1+z)^{-2}$ we have

$$
\kappa(t) \equiv 1 \quad \text { and } \quad c_{k}(t) \equiv(-1)^{k} \frac{2}{k} \quad \text { for } k=1,2, \ldots
$$

Hence the contribution $\Sigma(4 / k) \tau_{k}(t)$ in (3.9) cannot be changed if we want to obtain an estimate that is sharp for the Koebe function.

Now consider the properties of univalent functions used to prove $\varphi^{\prime}(t) \geqslant 0$. The proof uses Löwner's differential equation (3.3) only as it prescribes the motion of $c_{1}(t), c_{2}(t), \ldots, c_{n}(t)$ as given in the differential equation (3.6). Whether $c_{1}(t), \ldots, c_{n}(t)$ actually arise from a univalent function related to the choice of $\kappa(t)$ is not considered.

If we agree that only (3.6) is to be used to show $\varphi^{\prime}(t) \geqslant 0$, we are free to prescribe $c_{1}\left(t_{0}\right), \ldots, c_{n}\left(t_{0}\right)$ and a continuous $\kappa(t)$ with $|\kappa(t)|=1$. Then (3.6) has local solutions and the inequalities resulting from $\varphi^{\prime}\left(t_{0}\right) \geqslant 0$ give requirements of the functions $\tau_{k}$.

Let $\kappa(t) \equiv 1$ and, for some $L=1, \ldots, n$ and $0 \leqslant t_{0}<\infty$, let

$$
c_{k}\left(t_{0}\right)= \begin{cases}(-1)^{L}(2+\eta) / L & \text { for } k=L, \\ (-1)^{k} 2 / k & \text { for } k \neq L,\end{cases}
$$

where $\eta$ is a complex number of small magnitude.

Equation (3.6) then implies

$$
c_{k}^{\prime}= \begin{cases}0 & \text { for } k<L, \\ (-1)^{L} \eta & \text { for } k=L, \\ 2(-1)^{L} \eta & \text { for } k>L,\end{cases}
$$

where the argument $t_{0}$ is dropped. Hence

$$
\varphi^{\prime}=\sum_{k=1}^{n} \operatorname{Re}\left[2 \bar{c}_{k} c_{k}^{\prime}\right] \tau_{k}+\sum_{k=1}^{n}\left(k\left|c_{k}\right|^{2}-4 / k\right) \tau_{k}^{\prime} \geqslant 0
$$

implies that, as $\eta$ tends to zero,

$$
\operatorname{Re}\left[4 \eta \tau_{L}+\sum_{k=L+1}^{n} 8(-1)^{k+L} \eta \tau_{k}+4 \eta \frac{\tau_{L}^{\prime}}{L}\right]+O\left(|\eta|^{2}\right) \geqslant 0
$$

Since the argument of $\eta$ is arbitrary,

$$
\tau_{L}+2 \sum_{k=L+1}^{n}(-1)^{k+L} \tau_{k}+\frac{\tau_{L}^{\prime}}{L}=0 .
$$

If $L<n$, we also consider (5.4) for $L+1$ and add; we obtain

$$
\tau_{L}-\tau_{L+1}+\frac{\tau_{L}^{\prime}}{L}+\frac{\tau_{L+1}^{\prime}}{L+1}=0
$$

If $L=n$, then (5.5) follows at once from (5.4) where $\tau_{n+1}=0$. Now (5.5) is equivalent to the differential equation (2.2) of de Branges for $\left(t=t_{0}\right)$. The initial conditions $\tau_{k}(0)=n+1-k$ are specified by the inequality to be proved. Equation (2.2) and the initial conditions determine the function $\tau_{k}$ uniquely. 
Furthermore, $\varphi^{\prime} \geqslant 0$ implies each $\tau_{L}^{\prime} \leqslant 0$ at $t=t_{0}$. To show this fact we construct $c_{k}$ such that $\left|b_{k}+b_{k+1}+2\right|=0$ for $k \neq L$ and positive for $k=L$ and use (3.11).

(3) Knowing how to find the appropriate weight functions, we can consider whether the Bieberbach conjecture could be proved by the de Branges method applied directly. Let $f(z, t)=e^{t} z+a_{2}(t) z^{2}+a_{3}(t) z^{3}+\cdots$. We restrict to the case $n=3$ and look for real-valued weight functions $w_{1}(t)$ and $w_{2}(t)$ with $w_{1}(0)=0$ and $w_{2}(0)=1$ such that

$$
\psi(t)=\left|a_{2}\right|^{2} w_{1}(t)+\left|a_{3}(t)\right|^{2} w_{2}(t)-\left(4 w_{1}(t)+9 w_{2}(t)\right) e^{2 t}
$$

satisfies $\psi^{\prime}(t) \geqslant 0$ and $\psi(\infty)=0$. Note that the last term in (5.6) is determined by the requirement that $\psi(t) \equiv 0$ for $f(z, t)=e^{t} z(1+z)^{-2}$.

The proof that $\psi^{\prime} \geqslant 0$ should use only that

$$
a_{2}^{\prime}=2 a_{2}+2 \kappa(t) e^{t} \text { and } a_{3}^{\prime}=3 a_{3}+4 \kappa a_{2}+2 \kappa^{2} e^{t}
$$

If we use (5.6) and (5.7), it is easy to express $\psi^{\prime}$ as a polynomial in $a_{2}$ and $a_{3}$ :

$$
\psi^{\prime}=4\left|a_{2}\right|^{2} w_{1}+\left|a_{2}\right|^{2} w_{1}^{\prime}+\left(6\left|a_{3}\right|^{2}+\operatorname{Re} 8 \kappa \bar{a}_{3} a_{2}\right) w_{2}+\left|a_{3}\right|^{2} w_{1}^{\prime}+\cdots,
$$

where the remaining terms are of lower order. Consider $\kappa\left(t_{0}\right)=-1$ and $a_{2}\left(t_{0}\right)=2 x$ and $a_{3}\left(t_{0}\right)=3 x$ for a real variable $x$. From (5.8),

$$
\psi^{\prime}=\left(16 w_{1}+4 w_{1}^{\prime}+6 w_{2}+9 w_{2}^{\prime}\right) x^{2}+O(|x|) \text { as } x \rightarrow \infty .
$$

Since we can pick $x$ arbitrarily large, $\psi^{\prime}\left(t_{0}\right) \geqslant 0$ implies the coefficient of the leading term in (5.9) must be nonnegative.

On the other hand, making small variations from the Koebe function as in the previous remark, we deduce from $\psi^{\prime} \geqslant 0, w_{1}(0)=0$ and $w_{2}(0)=1$ that $w_{1}=6 e^{-3 t}$ $-6 e^{-4 t}$ and $w_{2}=e^{-4 t}$. Then for $t_{0}=0$, the coefficient of $x^{2}$ in (5.9) is -6 in contradiction to the conclusion that it must be nonnegative.

Hence $\left|a_{3}\right| \leqslant 3$ cannot be proved by this direct approach. What de Branges did (rewritten in the present context) was to consider

$$
\left|a_{2}\right|^{2} e^{-2 t} \tau_{1}+2\left|a_{3}-\frac{1}{2} a_{2}^{2} e^{-t}\right|^{2} e^{-2 t} \tau_{2}-\left(4 \tau_{1}+2 \tau_{2}\right)
$$

instead of (5.6). This gives $2\left|a_{2}\right|^{2}+2\left|a_{3}-\frac{1}{2} a_{2}\right|^{2} \leqslant 10$ which implies

$$
\left|a_{3}\right| \leqslant \frac{1}{2}\left|a_{2}\right|^{2}+\sqrt{5-\left|a_{2}\right|^{2}} \leqslant 3 \text {. }
$$

Clearly the Milin conjecture has motivated an appropriate combination of coefficients in expression (5.10).

ACKNowledgement. We are grateful to Professor S. Warschawski for his meticulous translation of the first Russian preprint of the de Branges proof. Also we appreciate communications from Professors D. Hamilton, J. Korevaar and G. Schober.

\section{REFERENCES}

1. R. Askey and G. Gaspar, Positive Jacobi polynomial sums. II, Amer. J. Math. 98 (1976), 709-737.

2. L. Bieberbach, Über die Koeffizienten derjenigan Potenzreichen, welche eine schlichte Abbildung des Einheitskreisses vermitteln, S.-B. Preuse. Akad. Wiss., Phys.-Math. K1. (1916), 940-955.

3. L. de Branges, $A$ proof of the Bieberbach conjecture, Preprint E-5-84, Steklov Math. Institute, LOMI, Leningrad, 1984, pp. 1-21. 
4.

5. Square summable power series, 2nd ed. (to appear).

6. P. L. Duren, Univalent functions, Springer-Verlag, New York, 1983.

7. G. Gasper, A short proof of an inequality used by de Branges in his proof of the Bieberbach, Robertson, and Milin conjectures (to appear).

8. K. Löwner (Charles Loewner), Untersuchungen über schlichte konforme Abbildungen des Einheitskreises, Math. Ann. 89 (1923), 103-121.

9. I. M. Milin, On the coefficients of univalent functions, Dokl. Akad. Nauk SSSR 176 (1967), 1015-1018; English transl., Soviet Math. Dokl. 8 (1967), 1255-1258.

10. __ Univalent functions and orthonormal systems, Moscow, 1971; English transl., Transl. Math. Monos., vol. 50, Amer. Math. Soc., Providence, R.I., 1977.

11. Ch. Pommerenke, Univalent functions, Vandenhoeck \& Ruprecht, Göttingen, 1975.

12. M. S. Robertson, A remark on odd schlicht functions, Bull. Amer. Math. Soc. 42 (1936), 366-370.

13. Quasi-subordination and coefficient conjectures, Bull. Amer. Math. Soc. 76 (1970), 1-9.

14. G. Szegö, Orthogonal polynomials, Amer. Math. Soc. Colloq. Publ., vol. 23, Amer. Math. Soc. Providence, R. I., 1959.

Department of Mathematics, University of California at San Diego, la Jolla, California 92093

Fachbereich Mathematik, Technische Universität, D-100 Berlin 12 